\title{
Dynamic endophenotypes and longitudinal trajectories: capturing changing aspects of development in early psychosis
}

\author{
Jai L. Shah, MD, MSc; M. Mallar Chakravarty, PhD; Ridha Joober, MD, PhD; Martin Lepage, PhD
}

The search for replicable markers - biological, psychological, social or their combinations - for psychiatric illnesses carries on. In psychosis, attention has been directed to at-risk and first-episode populations, given the possibility that following markers in young people will avoid the confounds of chronicity and exposure to pharmacological treatments. A variety of promising biomarkers have been evaluated, but few have passed through the rigorous screens of broader replication and demonstrated clinical utility. ${ }^{1,2}$

We argue in this editorial that such difficulties are unsurprising, given the construction of current studies and the fact that such disorders typically develop in adolescence and young adulthood. This "critical period" 3 for risk and onset of major youth mental illnesses, such as psychosis, coincides with deeply interlaced neurobiological, clinical, affective and cognitive development, not to mention profound changes in social interactions and exposures. We explore the implications of this backdrop, including how it should inform both the search for biomarkers and the design of future studies.

\section{Critical periods, neurobiology and mental health}

Adolescence to early adulthood, the age range when the early course of psychotic illness is most likely to emerge, is a window in which much is dynamic. At a neurobiological level, this includes neurons and synapses, neurotransmission, trophic factors and long-term potentiation, among other phenomena. ${ }^{4}$ However, it is also a period of sexual maturation, exposure to novel social and physical environments, different forms of stress, new models and contexts for learning, and developing cognitive abilities, reasoning and affective states. ${ }^{5}$ Whether adaptive or maladaptive, changes in brain structure and function are believed to occur in response to these internal and external stimuli and demands. For example, the frequency and type of stressful life events change as children enter adolescence; ${ }^{6}$ with these changes come further alterations in neurobiology that may denote increased sensitization to such stimuli. ${ }^{7}$ If more persistently dysregulated during a critical window, the long-term set-points of neurobiological processes or pathways may become distorted. Neurodevelopment that results from interactions between endogenous neurobiological elements and exogenous environmental factors could thus profoundly alter brain processes, leading to differential trajectories and clinical outcomes.

\section{Neurobiology, biomarkers and endophenotypes}

The acknowledgement that biological, psychological and social markers are not static but may themselves be subject to critical periods of influence or change, especially in youth mental health, suggests a path forward. Rather than relying on "snapshots" of data at a single time point to differentiate groups or pathology, it draws attention to the dynamic trajectories of individual markers and how they change. As individuals pass through periods of heightened risk, sequential evaluation of 1 or more markers could bring to light differential courses or inflection points that relate to specific clinical or functional outcomes.

The suggestion that neurodevelopmental alterations may cut across traditional psychiatric diagnostic schemas (such as DSM categories) $)^{8}$ dovetails with major contemporary neuroscience initiatives. In response to the perceived failure of symptom-based classification, the National Institute of Mental Health Research Domain Criteria (RDoC) were pioneered to "reimagine" classification systems agnostic of traditional diagnostic schema. ${ }^{9}$ To advance the study and treatment of mental illness, RDoC advocates argue that we must identify and focus on valence systems, cognition, social processing, and arousal and regulatory systems across multiple levels of analysis from genes and molecules to circuitry and (ultimately) behaviour. Similarly, the endophenotype concept has been articulated as a means of deconstructing unseen but measurable components "along the pathway" between genotype and syndromic illness. ${ }^{10}$ Endophenotypes are heritable traits that cosegregate with illness, but similar to RDoC categories can be

Correspondence to: J.L. Shah, Department of Psychiatry, PEPP-Montreal, Wilson Pavilion, 6875 LaSalle Blvd, Montreal, QC, Canada; jai.shah@ douglas.mcgill.ca.

DOI: 10.1503/jpn.160053 
explored at any level of analysis. While neither of these initiatives is without controversy, ${ }^{11,12}$ recent consortia in psychosis studies openly endorse either or both of them. . $^{13,14}$

In the context of youth mental health, RDoC- or endophenotype-based approaches could therefore shape our understanding of how psychobiological markers change over time in young people at varying degrees of risk for psychosis and link them to differential clinical, functional or other end points. Since the longitudinal trajectories of these markers may reveal more about pathogenesis and outcome than static ones, we argue that the focus should shift to the "dynamic" nature of endophenotypes and markers. Ultimately, it is likely that combinations of these markers measured longitudinally, not just single ones, will best map onto trajectories and outcomes. In the remainder of this editorial, we provide brief examples of 4 such markers and explore their implications for early psychosis.

\section{Dynamic endophenotypes and biomarkers}

\section{Structural neuroimaging}

The increasing use of longitudinal and repeated neuroimaging measures has allowed for the documentation of progressive brain changes in individuals with schizophrenia, reflecting the dynamic central nervous system. ${ }^{15-17}$ In a more homogeneous sample of patients with first-episode schizophrenia, Andreasen and colleagues ${ }^{18,19}$ found progressive grey and white matter loss in multiple regions over time related to the severity of psychotic symptoms, relapse duration and antipsychotic treatment intensity. The presence and persistence of negative symptoms in patients with schizophrenia and related psychoses is also associated with abnormalities in cortical thickness ${ }^{20}$ and white matter, ${ }^{21,22}$ cognitive deficits, and poor functional and vocational outcomes. ${ }^{23,24}$ Studies examining how putative endophenotypes interact and lead to differential effects on brain structure and functioning are therefore much needed. For example, in individuals at clinical high risk for psychosis, both negative symptoms and progressive cortical changes are frequently seen. ${ }^{25,26}$ This approach could derive clinical utility from imaging findings by enabling the early identification and targeted clinical follow-up of those likely to experience less favourable outcomes.

\section{Advanced imaging techniques}

Continuing innovations in neuroimaging ${ }^{27}$ have generated image processing techniques that capture salient alterations in brain structure at the group level ${ }^{28}$ and statistical techniques that are robust to uneven sampling among study participants. ${ }^{29}$ Longitudinal designs can thus improve statistical power for detecting group differences in neuroanatomical trajectories, allowing for the investigation of novel endophenotypes. For example, shape metrics are heritable and may have alterations specific to schizophrenia. ${ }^{30}$ Such methodologies provide insight into subcortical structures (such as the striatum) that are especially subject to confounding effects, or where standard volumetric comparisons fail to elucidate group differences. These metrics are also more sen- sitive to the progressive changes in neuroanatomy that characterize adolescent maturation. ${ }^{31}$ Advanced statistical, graph theory and community detection techniques are beginning to be used to infer underlying connectivity between brain regions, ${ }^{27}$ with adaptations of these methods to understand brain maturation. ${ }^{32,33}$ Future work will need to connect these different levels of observation into an integrated model. ${ }^{34} \mathrm{Ad}$ vanced imaging techniques could provide novel insight into a variety of microscopic-level alterations in neuroanatomy valuable indices for the study of progressive brain changes underlying the onset of psychosis. ${ }^{35-37}$

\section{Sleep alterations}

Among the most profound changes occurring during adolescence is a diminution in the amount of deep sleep: $\Delta(1-4 \mathrm{~Hz})$ electroencephalography power in nonrapid eye movement sleep declines massively during adolescence. ${ }^{38}$ It was on this basis that Feinberg ${ }^{39}$ originally advanced the neurodevelopmental hypothesis of schizophrenia, postulating that such alterations in sleep architecture correlate with synaptic pruning and maturation. Important changes in sleep patterns also take place during adolescence, notably a physiologic phase delay in the circadian rhythm. A dynamic defect in these maturational processes could be tracked over time and may offer another window to explore markers of risk for the emergence of psychotic symptoms. ${ }^{40}$ This is particularly relevant given emerging literature that sleep disorders are present in at-risk and first-episode phases of psychosis ${ }^{41-43}$ and are often early warning signs that precede the onset of illness or indicate relapse.

\section{Stress reactivity}

The hypothalamic-pituitary-adrenal axis harbours key markers of stress response and undergoes significant change during and after puberty. ${ }^{44}$ In psychosis, stress reactivity has been posited as a putative endophenotype owing to its cosegregation with illness and potential trait status in unaffected relatives. ${ }^{45}$ Well-designed laboratory-based paradigms have demonstrated short-term blunting of the dynamic cortisol response to stress, ${ }^{46,47}$ which may reflect exhaustion of the potential for a (healthy) robust phasic response to stress alongside the more tonic elevation found in at-risk populations. ${ }^{48}$ However, key questions remain about whether blunted stress response is a trait consistently found in unaffected family members and how the observed association between low tonic and robust phasic activity in healthy individuals changes along with longitudinal outcomes.

\section{Challenges and conclusions}

The examples we have discussed illustrate how putative endophenotypes could change dynamically over time - a phenomenon that would benefit from longitudinal studies. Such an approach presents 2 kinds of challenges. The first of these is concerned with theory and science: markers may diverge or change at different time points and rates; given 
this background variation, how and at what thresholds should risk for a particular trajectory be flagged or treated? Will change be expressed as linear, step-wise, cyclical, or in other forms? How can baseline states be indexed and/or maladaptive changes be determined in the context of already changing neurobiology - by age, phase of sexual maturation, clinical stage or otherwise? A necessary next step here is for within-subject changes in biomarkers to be correlated with longitudinal clinical/functional observations, making it plausible to weed out spurious correlations or to derive the presumably heterogeneous pathways to illness or resolution.

A second set of challenges relates to the clinical applicability of this approach. For individuals in early stages of mental distress or subclinical illness, it is likely that single biomarkers/ endophenotype trajectories will present as abnormal while an overall panel will remain ambiguous. Such a scenario should trigger closer monitoring and clinical correlation over time to either worsening or resolution of biomarkers and outcomes. In later (clinical high-risk or first-episode) stages, biomarker panels may become increasingly abnormal, prompting more intensive interventions with different risk/benefit profiles. It will therefore be important to identify and follow overlapping cohorts with varying degrees of enrichment for risk in order to understand whether putative endophenotypes better align with a priori risk groupings or evolving clinical/functional outcomes.

Finally, are youth mental health services capable of handling an integrative and coordinated approach given current resource constraints? At the research stage, intensive assaying at frequent time points is necessary. Ultimately, however, we envision the use of biomarkers not as a standard tool to be applied uniformly at great cost, but as a measured and targeted approach that can be selected to guide risk assessments or treatment decisions at key branch-points. And the economic arguments to fund "pre-emptive" clinical infrastructures are beginning to be made. ${ }^{49}$ Recent developments aimed at transforming youth mental health systems, ${ }^{50}$ including in Canada, ${ }^{51}$ will almost certainly involve at-risk populations beyond DSM-specific diagnoses. ${ }^{52}$ They now promise to form a scaffolding in which high-quality and researchinformed services ready to take up knowledge about dynamic endophenotypes can be situated.

Acknowledgments: We thank Matcheri Keshavan for early conversations that stimulated some of the ideas presented in this editorial.

Affiliations: From PEPP-Montréal, Douglas Mental Health University Institute (Shah, Chakravarty, Joober, Lepage); Cerebral Imaging Centre, Douglas Mental Health University Institute (Chakravarty); and the Department of Psychiatry, McGill University (Shah, Chakravarty, Joober, Lepage), Montreal, Que., Canada.

Competing interests: See jpn.ca/about for R. Joober. M. Lepage has received grants and personal fees from Otsuka/Lundbeck Alliance and Janssen Ortho and personal fees from Prophase. No other competing interests declared.

\section{References}

1. Kapur S. Looking for a "biological test" to diagnose "schizophrenia": Are we chasing red herrings? World Psychiatry 2011;10:32.
2. Lawrie SM, Olabi B, Hall J, et al. Do we have any solid evidence of clinical utility about the pathophysiology of schizophrenia? World Psychiatry 2011;10:19-31.

3. Birchwood M, Todd $\mathrm{P}$, Jackson $\mathrm{C}$. Early intervention in psychosis: the critical period hypothesis. Br J Psychiatry Suppl 1998;172:53-9.

4. Keshavan MS, Mehta UM, Padmanabhan JL, et al. Dysplasticity, metaplasticity, and schizophrenia: implications for risk, illness, and novel interventions. Dev Psychopathol 2015;27:615-35.

5. Millan MJ, Andrieux A, Bartzokis G, et al. Altering the course of schizophrenia: progress and perspectives. Nat Rev Drug Discov 2016;doi:10.1038/nrd.2016.28.

6. Gunnar M, Quevedo K. The neurobiology of stress and development. Annu Rev Psychol 2007;58:145-73.

7. Lupien SJ, McEwen BS, Gunnar MR, et al. Effects of stress throughout the lifespan on the brain, behaviour and cognition. Nat Rev Neurosci 2009;10:434-45.

8. McGorry P, Keshavan M, Goldstone S, et al. Biomarkers and clinical staging in psychiatry. World Psychiatry 2014;13:211-23.

9. Cuthbert BN. The RDoC framework: facilitating transition from ICD/DSM to dimensional approaches that integrate neuroscience and psychopathology. World Psychiatry 2014;13:28-35.

10. Gottesman II, Gould T. The endophenotype concept in psychiatry: etymology and strategic intentions. Am J Psychiatry 2003;160:636-45.

11. Joober R. On the simple and the complex in psychiatry, with reference to DSM 5 and research domain criteria. J Psychiatry Neurosci 2013;38:148-51.

12. Bracken $\mathrm{P}$, Thomas $\mathrm{P}$, Timimi $\mathrm{S}$, et al. Psychiatry beyond the current paradigm. Br J Psychiatry 2012;201:430-4.

13. Gur RE, Calkins ME, Gur RC, et al. The Consortium on the Genetics of Schizophrenia: neurocognitive endophenotypes. Schizophr Bull 2007;33:49-68.

14. Tamminga CA, Ivleva EI, Keshavan MS, et al. Clinical phenotypes of psychosis in the Bipolar-Schizophrenia Network on Intermediate Phenotypes (B-SNIP). Am J Psychiatry 2013;170:1263-74.

15. DeLisi LE. The concept of progressive brain change in schizophrenia: implications for understanding schizophrenia. Schizophr Bull 2008;34:312-21.

16. Hulshoff Pol HE, Kahn RS. What happens after the first episode? A review of progressive brain changes in chronically ill patients with schizophrenia. Schizophr Bull 2008;34:354-66.

17. Olabi B, Ellison-Wright I, McIntosh AM, et al. Are there progressive brain changes in schizophrenia? A meta-analysis of structural magnetic resonance imaging studies. Biol Psychiatry 2011;70:88-96.

18. Andreasen NC, Nopoulos P, Magnotta V, et al. Progressive brain change in schizophrenia: a prospective longitudinal study of firstepisode schizophrenia. Biol Psychiatry 2011;70:672-9.

19. Andreasen NC, Liu D, Ziebell S, et al. Relapse duration, treatment intensity, and brain tissue loss in schizophrenia: a prospective longitudinal MRI study. Am J Psychiatry 2013;170:609-15.

20. Bodnar M, Hovington CL, Buchy L, et al. Cortical thinning in temporo-parietal junction (TPJ) in non-affective first-episode of psychosis patients with persistent negative symptoms. PLoS One 2014;9:e101372.

21. Hovington $\mathrm{CL}$, Bodnar M, Chakravarty MM, et al. Investigation of white matter abnormalities in first episode psychosis patients with persistent negative symptoms. Psychiatry Res 2015;233:402-8.

22. Voineskos AN, Foussias G, Lerch J, et al. Neuroimaging evidence for the deficit subtype of schizophrenia. JAMA Psychiatry 2013;70:472-80.

23. Foussias G, Agid O, Fervaha G, et al. Negative symptoms of schizophrenia: clinical features, relevance to real world functioning and specificity versus other CNS disorders. Eur Neuropsychopharmacol 2014;24:693-709. 
24. Lepage M, Bodnar M, Bowie CR. Neurocognition: clinical and functional outcomes in schizophrenia. Can J Psychiatry 2014;59:5-12.

25. Piskulic D, Addington J, Cadenhead KS, et al. Negative symptoms in individuals at clinical high risk of psychosis. Psychiatry Res 2012;196:220-4.

26. Cannon TD, Chung $\mathrm{Y}, \mathrm{He} \mathrm{G}$, et al. Progressive reduction in cortical thickness as psychosis develops: a multisite longitudinal neuroimaging study of youth at elevated clinical risk. Biol Psychiatry 2015; 77:147-57.

27. Fitzsimmons J, Kubicki M, Shenton ME. Review of functional and anatomical brain connectivity findings in schizophrenia. Curr Opin Psychiatry 2013;26:172-87.

28. Emami S, Guimond S, Chakravarty M, et al. Cortical thickness and low insight into symptoms in enduring schizophrenia. Schizophr Res 2016;170:66-72.

29. Chakravarty MM, Rapoport JL, Giedd JN, et al. Striatal shape abnormalities as novel neurodevelopmental endophenotypes in schizophrenia: a longitudinal study. Hum Brain Mapp 2015;36:1458-69.

30. Roalf DR, Vandekar SN, Almasy L, et al. Heritability of subcortical and limbic brain volume and shape in multiplex-multigenerational families with schizophrenia. Biol Psychiatry 2015;77:137-46.

31. Raznahan A, Shaw PW, Lerch JP, et al. Longitudinal fourdimensional mapping of subcortical anatomy in human development. Proc Natl Acad Sci U S A 2014;111:1592-7.

32. Raznahan A, Lerch JP, Lee N, et al. Patterns of coordinated anatomical change in human cortical development: a longitudinal neuroimaging study of maturational coupling. Neuron 2011;72:873-84.

33. Alexander-Bloch AF, Reiss PT, Rapoport J, et al. Abnormal cortical growth in schizophrenia targets normative modules of synchronized development. Biol Psychiatry 2014;76:438-46.

34. Keshavan MS, Giedd J, Lau JYF, et al. Changes in the adolescent brain and the pathophysiology of psychotic disorders. Lancet Psychiatry 2014;1:549-58.

35. Zhang H, Schneider T, Wheeler-Kingshott CA, et al. NODDI: practical in vivo neurite orientation dispersion and density imaging of the human brain. Neuroimage 2012;61:1000-16.

36. Waehnert MD, Dinse J, Schäfer A, et al. A subject-specific framework for in vivo myeloarchitectonic analysis using high resolution quantitative MRI. Neuroimage 2016;125:94-107.

37. Stikov N, Campbell JSW, Stroh T, et al. In vivo histology of the myelin g-ratio with magnetic resonance imaging. Neuroimage 2015; 118:397-405.

38. Feinberg I, Campbell IG. Sleep EEG changes during adolescence: an index of a fundamental brain reorganization. Brain Cogn 2010;72:56-65.
39. Feinberg I. Schizophrenia: Caused by a fault in programmed synaptic elimination during adolescence? J Psychiatr Res 1982-83;17:319-34.

40. Lunsford-Avery JR, LeBourgeois MK, Gupta T, et al. Actigraphicmeasured sleep disturbance predicts increased positive symptoms in adolescents at ultra high-risk for psychosis: a longitudinal study. Schizophr Res 2015;164:15-20.

41. Zanini M, Castro J, Coelho FM, et al. Do sleep abnormalities and misaligned sleep/circadian rhythm patterns represent early clinical characteristics for developing psychosis in high risk populations? Neurosci Biobehav Rev 2013;37:2631-7.

42. Lunsford-Avery JR, Orr JM, Gupta T, et al. Sleep dysfunction and thalamic abnormalities in adolescents at ultra high-risk for psychosis. Schizophr Res 2013;151:148-53.

43. Keshavan MS, Diwadkar VA, Montrose DM, et al. Premorbid characterization in schizophrenia: the Pittsburgh High Risk Study. World Psychiatry 2004;3:163-8.

44. Walker E, Mittal V, Tessner K. Stress and the hypothalamic pituitary adrenal axis in the developmental course of schizophrenia Annu Rev Clin Psychol 2008;4:189-216.

45. Lataster J, Collip D, Ceccarini J, et al. Familial liability to psychosis is associated with attenuated dopamine stress signaling in ventromedial prefrontal cortex. Schizophr Bull 2014;40:66-77.

46. Dickerson SS, Kemeny ME. Acute stressors and cortisol responses: a theoretical integration and synthesis of laboratory research. Psycho Bull 2004;130:355-91.

47. Jones SR, Fernyhough C. A new look at the neural diathesis-stress model of schizophrenia: the primacy of social-evaluative and uncontrollable situations. Schizophr Bull 2007;33:1171-7.

48. Shah JL, Malla AK. Much ado about much: stress, dynamic biomarkers and HPA axis dysregulation along the trajectory to psychosis. Schizophr Res 2015;162:253-60.

49. Brimblecombe N, Knapp M, Murguia S, et al. The role of youth mental health services in the treatment of young people with serious mental illness: 2-year outcomes and economic implications. Early Interv Psychiatry 2015; doi: 10.1111/eip.12261.

50. Cross SPM, Hermens DF, Scott EM, et al. A clinical staging model for early intervention youth mental health services. Psychiatr Serv 2014;65:939-43.

51. Iyer S, Boksa P, Lal S, et al. Transforming youth mental health: a Canadian perspective. Ir J Psychol Med 2015;32:51-60.

52. Shah JL. Sub-threshold mental illness in adolescents: within and beyond DSM's boundaries. Soc Psychiatry Psychiatr Epidemiol 2015; 50:675-7. 